\title{
Solubilization of Unilamellar Liposomes by Betaine-Type Zwitterionic/Anionic Surfactant Systems
}

\author{
A. de la Maza* and J.L. Parra \\ Departamento de Tensioactivos, Centro de Investigación y Desarrollo (C.I.D.), \\ Consejo Superior de Investigaciones Cientiticas (C.S.I.C.), 08034 Barcelona, Spain
}

\begin{abstract}
The mechanisms governing the solubilizing interactions between zwitterionic/anionic mixed surfactant systems at different molar fractions of the zwitterionic surfactant $\left(X_{z w i t t e r}\right)$ and neutral or electrically charged unilamellar liposomes were investigated. The mixed systems were formed by $\mathrm{N}$-dodecyl-N, $\mathrm{N}$-dimethylbetaine and sodium dodecyl sulfate in the presence of piperazine-1,4-bis-(2-ethanesulfonic acid) buffer at $\mathrm{pH}$ 7.20. Unilamellar liposomes formed by egg phosphatidylcholine, in some cases together with stearylamine or phosphatidic acid, were used. solubilization was detected as a decrease in static light-scattering of liposomes. Two parameters were regarded as corresponding to the effectivesurfactant/lipid molar ratios $(\mathrm{Re})$ at which the surfactant system (i) saturated the liposomes, Re,", and (ii) led to a total solubilization of liposomes, Re,,. From these parameters the bilayer/aqueous medium surfactant partition coefficients for the saturation $\left(\mathrm{K}_{\mathrm{sat}}\right)$ and complete bilayer solubilization $\left(K_{\mathrm{sol}}\right)$ were determined. When $X_{z \text { witter }}$ was 0.40 , The Re and $K$ parameters showed a maximum, whereas the critical micellar concentration (CMC) of these systems exhibited a minimum, regardless of the electrical charge of bilayers. Given that the ability of the surfactant systems to saturate or solubilize liposomes is inversely related

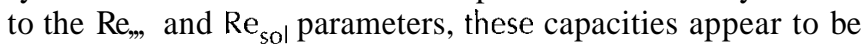
directly correlated with the CMC of the mixed systems. The similarity of both $K_{\text {sat }}$ and $K_{\text {sol }}$ (particularly for $X_{z w i t t e r}=0.2-0.8$ ) suggests that a similar partition equilibrium governs both the saturation and the complete solubilization of bilayers, the free surfactant concentration $\left(\mathrm{S}_{\mathrm{a}^{\prime}} \mathrm{S}_{\mathrm{b}}\right)$, remaining almost constant with similar values to the CMC for each mixed system studied.

JAOCS 72, 131-136 (19951.
\end{abstract}

KEY WORDS: Bilayer/aqueous medium surfactant partition coefficients, critical micelle concentration, effective surfactant/phospholipid molar ratios, interaction liposome/(dodecyl betaine/sodium dodecyl sulfate) mixed systems, light (PL) scattering changes and liposomes.

The interaction of surfactants with phospholipid (PL) bilayers in excess water leads to the breakdown of lamellar structures and to the formation of lipid-surfactant mixed micelles $(1,2)$. This process is commonly denoted as "solubilization." Many studies have been devoted to understanding the princi-

\footnotetext{
* To whom correspondence should be addressed at: Departamento de Tensioactivos. Centro de Investigación y Desarrollo (C.I.D.). Jordi Girona, 18 26. 08034 Barcelona. Spain.
}

ples that govern this complex process (3-9). A significant contribution has been made by Lichtenberg (10), who postulated that the critical effective surfactant/lipid ratio that produces solubilization depends on the surfactant critical micellar concentration (CMC) and on the bilayer/aqueous medium distribution coefficients $(K)$, rather than on the nature of the surfactants. Urbaneja $\boldsymbol{e}$ al. (11) demonstrated that when performed systematically, light-scattering measurements constitute a convenient technique for the quantitative study of bilayer solubilization by surfactants.

Zwitterionic surfactants have a strong interaction or complex formation with ionic surfactants in aqueous solutions $(12,13)$. Betaines, which are capable of accepting a proton, interact much more strongly with anionic than with cationic surfactants. These interactions lead to mixed surfactant solution systems that show greater surface activity than that attainable with any of the individual surfactants of the mixture at the same concentration and, consequently. exhibit synergism. Thus, zwitterionic surfactants have been used as boosters of several anionic surfactants in industrial applications, and their mixed properties have also been reported $(14,15)$. The effect of the micellar solution phase of these surfactant mixtures in avoiding or at least in reducing the level of anionic/protein interaction has been suggested by several investigators as a way of slowing down the irritation potential of anionic surfactants (16-18).

In recent papers. we reported studies on the subsolubilizing interactions of the $\mathrm{N}$-dodecyl-N,N-dimethylbetaine $\left(\mathrm{C}_{12^{-}}\right.$ Bet)/sodium dodecyl sulfate (SDS) systems with unilamellar liposomes and the solubilizing interactions of these two surfactants when interacted individually with these liposomic structures $(19,20)$. In the present work we seek to extend these investigations by characterizing the solubilization of liposomes by mixtures of these surfactants to correlate the interesting synergism that exists between these two types of surfactants and their solubilizing capacity when applied to phosphatidylcholine (PC) unilamellar vesicles. The solubilization process was assessed as a decrease in the light scattered by the liposome/surfactant systems. To evaluate the light-scattering variations, two parameters were determined, namely $\mathrm{Re},$, , and $\mathrm{Re},,$, according to the three-stage model adopted by Lichtenberg $\boldsymbol{e t}$ al. (2.10). The knowledge of the 
different phases involved in these solubilizing interactions could be useful in improving our understanding of these synergisms and in establishing a criterion for the evaluation of the activity of these mixtures with respect to biological membranes.

\section{EXPERIMENTAL PROCEDURES}

Materials. PC was purified from egg lecithin at Merck (Darmstadt, Germany) according to the method of Singleton et al. (21) and was pure by thin-layer chromatography (TLC). Phosphatidic acid (PA) from egg yolk lecithin and stearylamine (SA) were purchased from Sigma Chemical Co. (St. Louis, MO). Both lipids were stored in chloroform under nitrogen at $-20^{\circ} \mathrm{C}$ until use.

$\mathrm{C}_{12}$-Bet was specially prepared by Albright and Wilson, Ltd. (Warley, West Midlands, United Kingdom); the active matter was $30 \%$ in aqueous solution and the free amino content was $0.20 \%$. SDS was obtained from Merck and further purified by a column chromatographic method (22). Piperazine-1,4-bis-(2-ethanesulfonic acid) (PIPES buffer) obtained from Merck was prepared as $20 \mathrm{mM}$ PIPES adjusted to $\mathrm{pH}$ 7.20 with $\mathrm{NaOH}$; it contained $110 \mathrm{mM} \mathrm{Na}_{2} \mathrm{SO}_{4}$. Water was purified by the Milli-Ro system (Millipore. Milford, MA). Polycarbonate membranes and membrane holders were purchased from Nucleopore (Pleasanton, CA).

Liposome preparation. Unilamellar liposome vesicles of a defined size (about $100 \mathrm{~nm}$ ) were prepare by extrusion of large unilamellar vesicles previously obtained by the reversephase evaporation method $(23,24)$ based on an earlier method described by Szoka and Papahadjopoulos (25). A lipid was formed by removing the organic solvent by rotatory evaporation from a chloroform solution of lipids (lipid composition PC or PC/PA, PC/SA 9:1 molar ratio). The lipids were then redissolved in diethyl ether, and the PIPES buffer was added to the solution of lipids. Gentle sonication led to the formation of a water-in-oil-type emulsion. After evaporating the ethyl ether under reduced pressure, a viscous gel was formed. The elimination of the final traces of organic solvent at high vacuum transformed the gel into a liposome suspension in which no traces of ether were detectable by nuclear magnetic resonance (NMR). Ether was washed in advance with the PIPES buffer and stored in bottles over water-containing bisulfite to obtain total elimination of ether in the liposome suspensions (26). Unilamellar vesicles were obtained by extrusion of vesicle suspensions through 800, 400, 200 and 100 $\mathrm{nm}$ polycarbonate membranes to achieve a uniform size distribution (27). The range of PL concentration in liposome suspension studied was $0.5-5.0 \mathrm{mM}$.

Determination of lipid bilayer concentration. The lipid bilayer concentration of the liposome suspensions after preparation was determined by TLC coupled to an automated flame-ionization detection system (Iatroscan MK-5; Iatron Lab. Inc. Tokyo, Japan) (28).

Determination of particle size distribution and stability of liposome preparations. The mean size and polydispersity of the liposome suspensions were determined with a photon correlator spectrometer (Malvern Autosizer 4700c PS/MV; Malvern, England). The particle size distributions were established by particle number measurement. The samples were adjusted to the appropriate concentration range with PIPES buffer, and the measurements were taken at $25^{\circ} \mathrm{C}$ at a reading angle of $90^{\circ}$.

Solubilizing parameters. The perturbation produced by the surfactants in the PL bilayers leads to the solubilization of the lipid components via mixed micelle formation (10). This solubilization results in changes in light scattering of these systems, which depend on the nature of both surfactant and lipid components. This can be monitored by measuring the variations in light scattering during the solubilizing processes $(11,29)$.

When defining the parameters related to the solubilization of liposomes, it is essential to consider that the mixing of lipids and surfactants is not ideal due to the specific interactions between both components. which has been demonstrated for a variety of amphiphiles $(30,31)$. To evaluate the alterations caused by the $C_{12}$ Bet/SDS surfactant mixed systems on lipid bilayers, the effective surfactant/PL molar ratio $R e$ in an aggregate (liposome or micelle) is defined as follows (10):

$$
R e=\frac{[\text { total surfactant }]-[\text { surfactant monomer }]}{[\text { total PL }]-[\mathrm{PL} \text { monomer }]}
$$

The second term of the denominator is negligible, due to the low solubility of PL (PL concentration $[\mathrm{mM}]$ ) in water. Likewise, it is generally admitted that an equilibrium partition of surfactants between bilayer and the aqueous medium governs the incorporation of surfactants into liposomes. thereby producing saturation and solubilization of these structures.

In the analysis of the equilibrium partition model proposed by Schurtenberger (32) for bile salt/lecithin systems, Lichtenberg (10) and Almog et al. (6) have shown that. for a mixing of lipids [at a concentration PL $(\mathrm{mM})$ ] and surfactant [at a concentration $S_{T}(\mathrm{mM})$ ], in dilute aqueous media, the distribution of surfactant between lipids bilayers and aqueous media obeys a partition coefficient $K$, given (in $\mathrm{mM}^{-1}$ ) by:

$$
K=\frac{S_{B}}{\left[\mathrm{PL}+S_{B}\right\rceil \cdot S_{w}}
$$

where $S_{B}$ is the concentration of surfactant in the bilayers $(\mathrm{mM})$ and $S_{W}$ is the surfactant concentration in the aqueous medium (mM). For PL $\gg S_{B}$, the definition of $K$, as given by Schurtenberger, applies:

$$
K=\frac{S_{B}}{\left(\mathrm{PL} \cdot S_{W}\right)}=\frac{R e}{S_{W}}
$$

whereas $R e$ is the abovementioned ratio of surfactant to PL in the vesicle bilayer: $\left(\operatorname{Re}=S_{B} / \mathrm{PL}\right)$. Under any other conditions, 
Equation 2 has to be employed to define $K$; this yields:

$$
K=\frac{R e}{S_{W}[1+R e]}
$$

This approach is consistent with the experimental data offered by Lichtenberg (10) and Almog (6) for different surfactant PL mixtures over wide ranges of $R e$ values. Given that the range of PL concentrations used in our investigation is similar to that used by Almog to test his equilibrium partition model, the $\mathrm{K}$ parameter has been determined with this equation.

The overall solubilization process of PL bilayers by surfactants can be characterized by two parameters, termed $R e$,, and $R e_{\text {sol }}$, according to the nomenclature adopted by Lichtenberg $(2,10)$, corresponding to the surfactant/lipid molar ratios at which light scattering starts to decrease and shows no further decrease. These parameters correspond to the $R e$ at which the surfactant (i) saturates the liposomes and (ii) leads to total solubilization of the liposomes. The determination of these parameters can be carried out on the basis of the linear dependence that exists between the surfactant concentrations that are required to achieve these parameters and the PL concentration in liposomes. The equations describing the surfactant concentration needed to satuate the bilayer (Equation 5) or to achieve the complete solubilization of liposomes via mixed micelles (Equation 6) are given as:

$$
\begin{gathered}
S_{T}=S_{a}+R e_{s u t} \bullet[\mathrm{PL}] \\
S_{T}=S_{b}+R e_{s o l} \bullet[\mathrm{PL}]
\end{gathered}
$$

whereas the $R e_{\text {sat }}$ or $R e_{\text {,, }}$ and the aqueous concentrations of surfactants $\left(\mathrm{S}\right.$, and $\left.S_{b}\right)$ are, in each curve, respectively, the slope and the ordinate, at the origin (zero PL concentration).

From these parameters, the partition coefficients of surfactant corresponding to the saturation $K_{\text {sat }}$ and total solubilization $K_{\text {sol }}$ of liposomes have been determined by applying Equation 4. This partition coefficient during solubilization may be regarded as a dynamic equilibrium between the different transition structural stages from lipid bilayers to mixed micelles.

Liposome suspensions were adjusted to the proper lipid concentration (from 1.0 to $10.0 \mathrm{mM}$ ). Equal volumes of the appropriate $\mathrm{C}_{\mathrm{I}}$,-BetISDS surfactant mixed solutions were added, and the resulting mixtures were left to equilibrate for $24 \mathrm{~h}$. Light-scattering measurements were made at $25^{\circ} \mathrm{C}$ with a Shimadzu (Tokyo, Japan) RF-540 spectrofluorophotometer equipped with a thermoregulated cell compartment, with both monochromators adjusted to $500 \mathrm{~nm}$. The assays were carried out in triplicate, and the results given are the average of those obtained.

\section{RESULTS AND DISCUSSION}

Determination of particle size distribution and stability of liposome preparations. The particle size distribution of the liposome suspensions after preparation (PL concentration rang- ing from 0.5 to $5.0 \mathrm{mM}$ ) varied little (around $100 \mathrm{~nm}$ ). The polydispersity indices were lower than 0.1 , indicating that the size distributions were homogeneous. Furthermore, the particle size distribution of liposomes after the addition of equal volumes of PIPES buffer and equilibration for $24 \mathrm{~h}$ at $25^{\circ} \mathrm{C}$ showed in all cases values that were similar to those obtained after preparation, with a slight increase in the polydispersity index (from 0.12 to 0.14 ). Hence, the liposome preparations appeared to be reasonably stable in the absence of surfactants under the experimental conditions used in the liposome solubilization studies.

Solubilization studies. To determine the solubilizing capacity of the $\mathrm{C}_{12}$-Bet/SDS mixed systems on neutral and electrically charged unilamellar liposomes, a series of experiments were carried out to study the disrupting effect caused by these mixed systems at different molar fractions of the zwitterionic surfactant $\left(X_{\text {zwitter }}\right)$.

The solubilization process was studied by monitoring the variation in the light scattered by the surfactant/liposome systems as a function of surfactant concentration. In accordance with the procedure described by Urbaneja et al. (11), changes in the light scattered by these systems were determined $24 \mathrm{~h}$ after the addition of surfactants to liposomes at $25^{\circ} \mathrm{C}$. The lipid bilayers consisted of PC unilamellar vesicles, to which PA or SA was added, yielding liposomes with molar ratios $\mathrm{PC} / \mathrm{PA}$ or PC/SA of 9:1 to increase the negative or positive charge of the bilayers.

Figure 1 shows the percentage change of light scattering that corresponded to the solubilization curves of neutral liposome preparations (lipid concentration from 0.5 to $5.0 \mathrm{mM}$ ) arising from the addition of different concentrations of surfactant mixed systems for $X^{*}=0.4$. From these curves, the surfactant concentration that produced the saturation $\left(S_{s a t}\right)$ and the total solubilization of the liposomes $\left(S_{s o l}\right)$ can be obtained by graphical methods. The arrows A and B (curve for

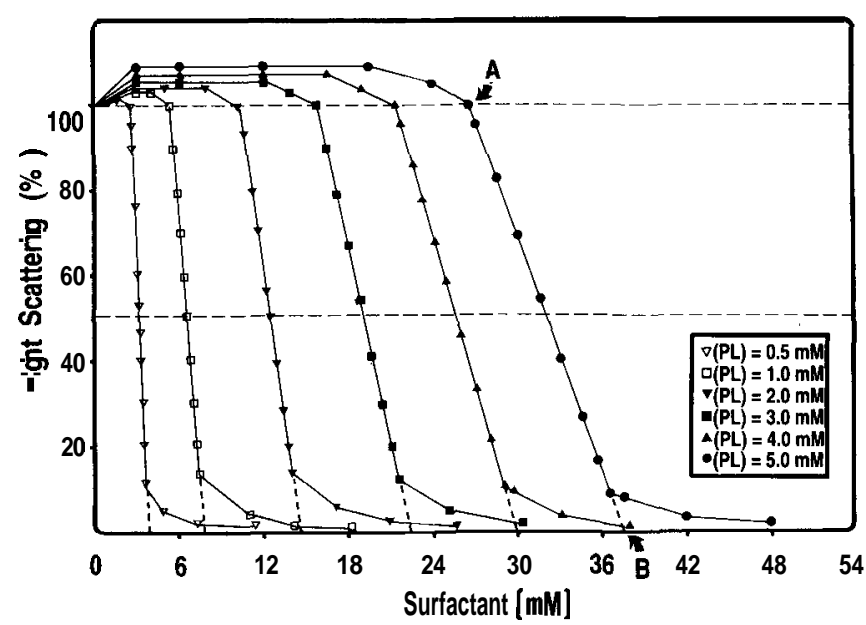

FIG. 1. Percentage change in light scattering of neutral unilamellar liposomes (the bilayer lipid concentration ranging from 0.5 to $5.0 \mathrm{mM}$ ) vs. concentration of the $\mathrm{N}$-dodecyl-N, N-dimethylbetaine/sodium dodecyl sulfate mixed surfactant system for the molar fraction of the zwitterionic surfactant $=0.4$. 


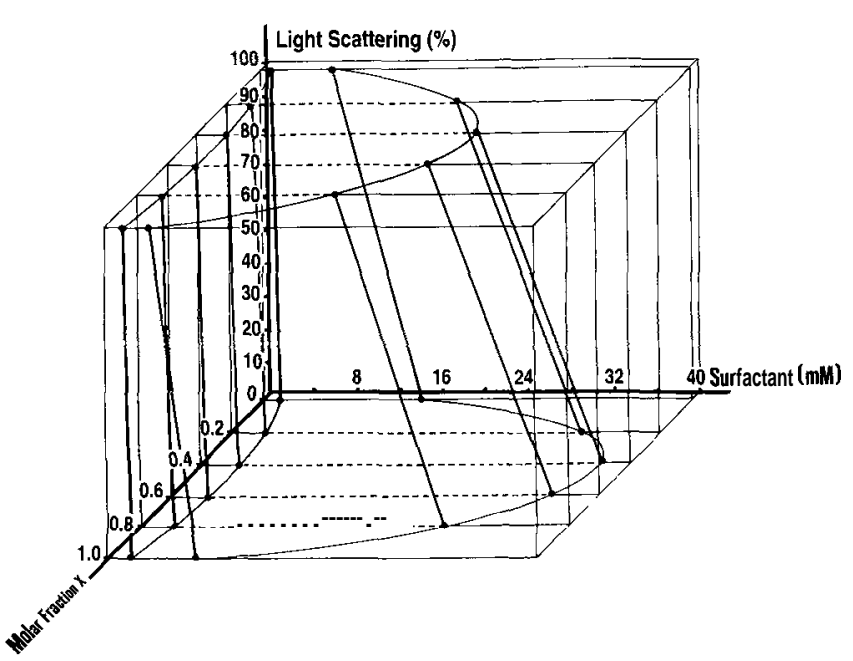

FIC. 2. Percentage change in light scattering of neutral liposomes (bilayer lipid concentration $0.5 / 5.0 \mathrm{mM}$ ) vs. concentration of $\mathrm{N}$-dodecyl$\mathrm{N}, \mathrm{N}$-dimethylbetaine/sodium dodecyl sulfate mixed surfactant systems for different molar fractions of the zwitterionic surfactant.

lipid concentration $5.0 \mathrm{mM}$ ) correspond to these parameters. When plotting the extrapolation of these solubilization curves (lipid concentration $0.5-5.0 \mathrm{mM}$ ) vs. the surfactant concentration for different molar fractions of the zwitterionic surfactant, a three-dimensional picture is obtained (Fig. 2). The surfactant concentration needed to achieve the saturation or solubilization of liposomes shows a maximum value for the $X_{\text {zwitter }}=0.4$.

Plotting the $S_{s a t}$ and $S_{s o l}$ concentrations, previously obtained at different $X_{\text {zwitter }}$, vs. PL concentration, Figure 3, shows the straight lines corresponding to neutral liposomes. An acceptable linear relationship is established in each case. The straight lines obtained correspond to the aforementioned Equations 5 and 6 from which $R e$. K and $S_{a}$ and $S_{b}$ were determined. Similar results were obtained when treating electrically charged liposomes (PC/PA or PCISA, 9:1 molar ratio) with these systerns under the same conditions (results not shown). The Re and $\mathrm{K}$ parameters obtained, including the regression coefficients of the straight lines and the CMC values of the single surfactants and the mixed systems in the buffered medium previously reported (19), are shown in Table 1.

In the vast majority of cases, solubilization of liposomes was virtually unaffected by the electric charge of the lipid bilayers, particularly in the range of $X_{\text {zwitter }}$ from 0.4 to 0.6. These results are surprising, given that the association of these surfactants in the surfactant mixed systems occurs by electrostatic attraction between the cationic portion of the betaine and the dodecyl sulfate ion (33). Frorn these findings, we may assume that the nonpolar forces, especially those that are hydrophobic in nature, may play an important role in the interaction of these surfactant mixed systems with lipid bilayers, which leads to the saturation and subsequent solubilization of these structures via mixed micelle formation. These results are in agreement with those previously reported by Urbarieja et al. (11), with respect to the role of the hydrophobic effect in the solubilization of liposomes by differ-
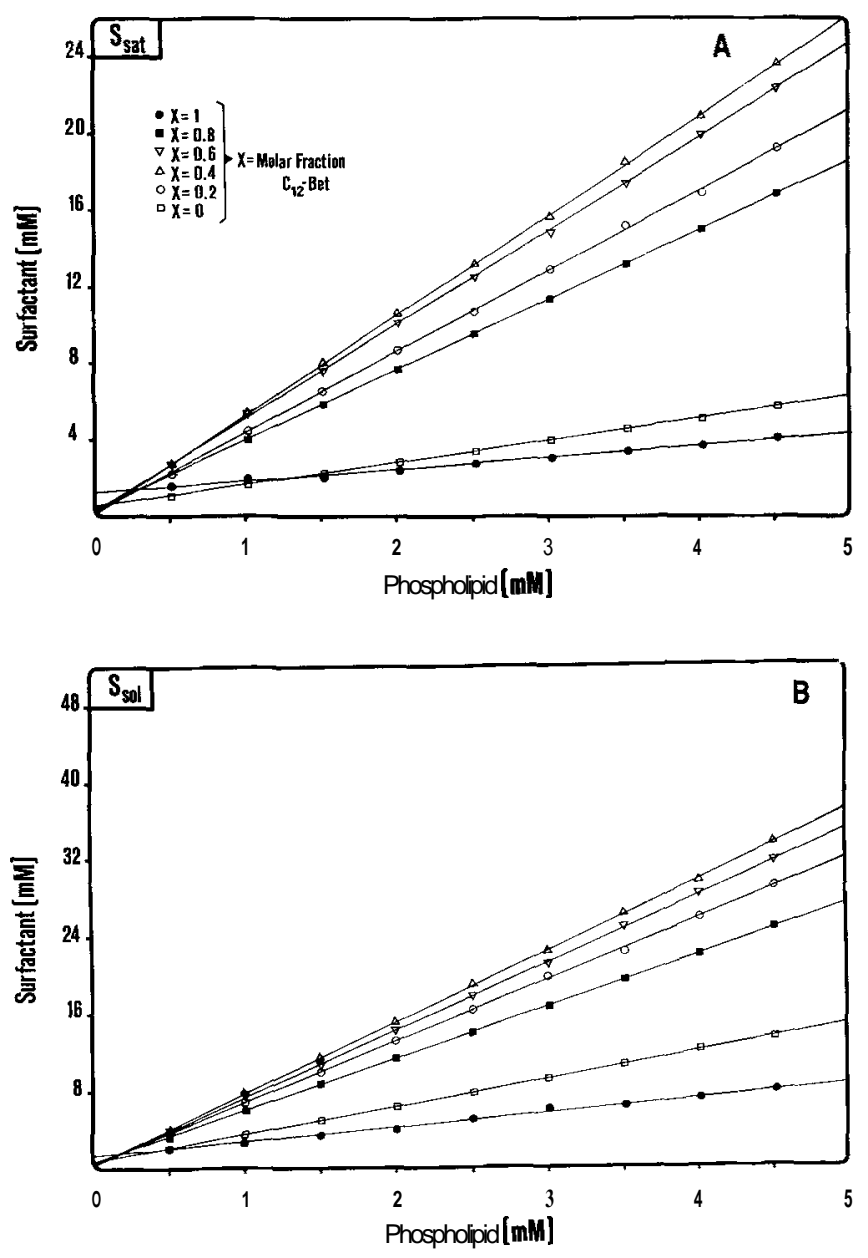

FIC. 3. Concentration of the $\mathrm{N}$-dodecyl-N, N-dimethylhetaine/sodium dodecyl sulfate surfactant systems for different inolar fractions ot the zwitterionic surfactant corresponding to saturation $\left(S_{\text {sat }}\right)$ and solubilization $\left(S_{s o l}\right)$ ot neutral liposomes vs. bilayer lipid concentration.

ent single surfactants. Furthermore, the Re and $\mathrm{K}$ values increased as the molar fraction of the zwitterionic surfactant rose. showing a maximum for $X_{\text {zxitter }}=0.4$ regardless of the electrical charge of lipid bilayers. This $X_{\text {zwitter }}$ corresponded to the minimum activity of the systems, given that the $\boldsymbol{R} \boldsymbol{e}$ value decreases as the surfactant activity increases both in the saturation and solubilization of lipid bilayers. The variation of $R e_{\text {sutt }}$ and $R e_{s o l}$ parameters vs. $X_{\text {zwitter }}$ for neutral liposomes is plotted in Figure 4.

Moreover, the surfactant concentration in the aqueous medium was always comparable to the $\mathrm{CMC}$ of each surfactant system tested. These results support the generally admitted assumption that the concentration of free surfactant must reach the CMC for solubilization to occur (10).

As for the partition coefficients, it is noteworthy that these parameters for saturation $\left(K_{\text {sat }}\right)$ and complete solubilization of bilayers $\left(K_{s o l}\right)$ achieved similar values, particularly in the range of $X_{\text {zwitter }}$ from 0.2 to 0.8 . These findings suggest that a similar partition equilibrium governs both the incorporation of surfactant molecules into the lipid bilayers and the association of the surfactant mixed molecules with the lipid-building liposomes to form mixed micelles. This similar partition 
TABLE 1

Solubilizing Parameters (Re and $K$ ) of Neutra1 and Electrically Charged Liposomes Treated with the Mixed Surfactants Systems N-Dodecyl-N, N-Dimethylbetaine $\left(\mathrm{C}_{12}\right.$-Bet)/SDS for Different Molar Fractions of the Zwitterionic Surfactant $\left(X_{\text {zwitter }}\right)$ a

\begin{tabular}{|c|c|c|c|c|c|c|c|c|c|}
\hline & $\begin{array}{l}\text { C12-Bet } \\
\left(X_{\text {zwitter }}\right)\end{array}$ & $\begin{array}{l}C M C \\
(\mathrm{mM})\end{array}$ & $S_{a}$ & $S_{b}$ & $R e_{. . .}$ & $R e_{s o l}$ & $K_{\mathrm{sat}}$ & $K_{\text {sol }}$ & $r^{2}$ \\
\hline $\mathrm{PC} / \mathrm{PA}$ & 0 & 0.50 & 0.49 & 0.52 & 1.18 & 2.84 & 1.10 & 1.42 & 0.992 \\
\hline \multirow[t]{5}{*}{$(9: 1)$} & 0.2 & 0.22 & 0.22 & 0.24 & 4.26 & 6.40 & 3.68 & 3.60 & 0.997 \\
\hline & 0.4 & 0.16 & 0.16 & 0.17 & 5.30 & 7.44 & 5.25 & 5.18 & 0.994 \\
\hline & 0.6 & 0.21 & 0.21 & 0.22 & 4.97 & 7.0 & 3.96 & 3.97 & 0.996 \\
\hline & 0.8 & 0.41 & 0.41 & 0.44 & 3.68 & 5.42 & 1.91 & 1.91 & 0.995 \\
\hline & 1.0 & 1.25 & 1.25 & 1.30 & 0.60 & 1.40 & 0.30 & 0.44 & 0.996 \\
\hline \multirow[t]{6}{*}{ Egg PC } & 0 & 0.50 & 0.50 & 0.53 & 1.10 & 2.70 & 1.04 & 1.37 & 0.992 \\
\hline & 0.2 & 0.22 & 0.21 & 0.23 & 4.20 & 6.30 & 3.84 & 3.75 & 0.994 \\
\hline & 0.4 & 0.16 & 0.15 & 0.16 & 5.31 & 7.45 & 5.61 & 5.51 & 0.998 \\
\hline & 0.6 & 0.21 & 0.20 & 0.21 & 4.98 & 7.05 & 4.16 & 4.17 & 0.999 \\
\hline & 0.8 & 0.41 & 0.40 & 0.42 & 3.70 & 5.45 & 1.96 & 2.01 & 0.991 \\
\hline & 1.0 & 1.25 & 1.27 & 1.32 & 0.63 & 1.43 & 0.30 & 0.44 & 0.993 \\
\hline PCISA & 0 & 0.50 & 0.50 & 0.50 & 1.10 & 2.68 & 1.04 & 1.45 & 0.989 \\
\hline \multirow[t]{5}{*}{$(9: 1)$} & 0.2 & 0.22 & 0.22 & 0.23 & 4.20 & 6.28 & 3.67 & 3.75 & 0.992 \\
\hline & 0.4 & 0.16 & 0.15 & 0.16 & 5.32 & 7.43 & 5.61 & 5.51 & 0.996 \\
\hline & 0.6 & 0.21 & 0.20 & 0.21 & 4.96 & 7.05 & 4.16 & 4.17 & 0.992 \\
\hline & 0.8 & 0.41 & 0.40 & 0.43 & 3.78 & 5.49 & 1.97 & 1.96 & 0.993 \\
\hline & 1.0 & 1.25 & 1.28 & 1.34 & 0.66 & 1.48 & 0.31 & 0.44 & 0.997 \\
\hline
\end{tabular}

${ }^{a}$ The critical micellar concentration (CMC) values of the single surfactants and the surfactant mixed systems (Ref. 19), as well as the regression coefficients of the straight lines obtained are also included. PC, phosphatidylcholine; PA, phosphatidic acid; SA, stearylamine.

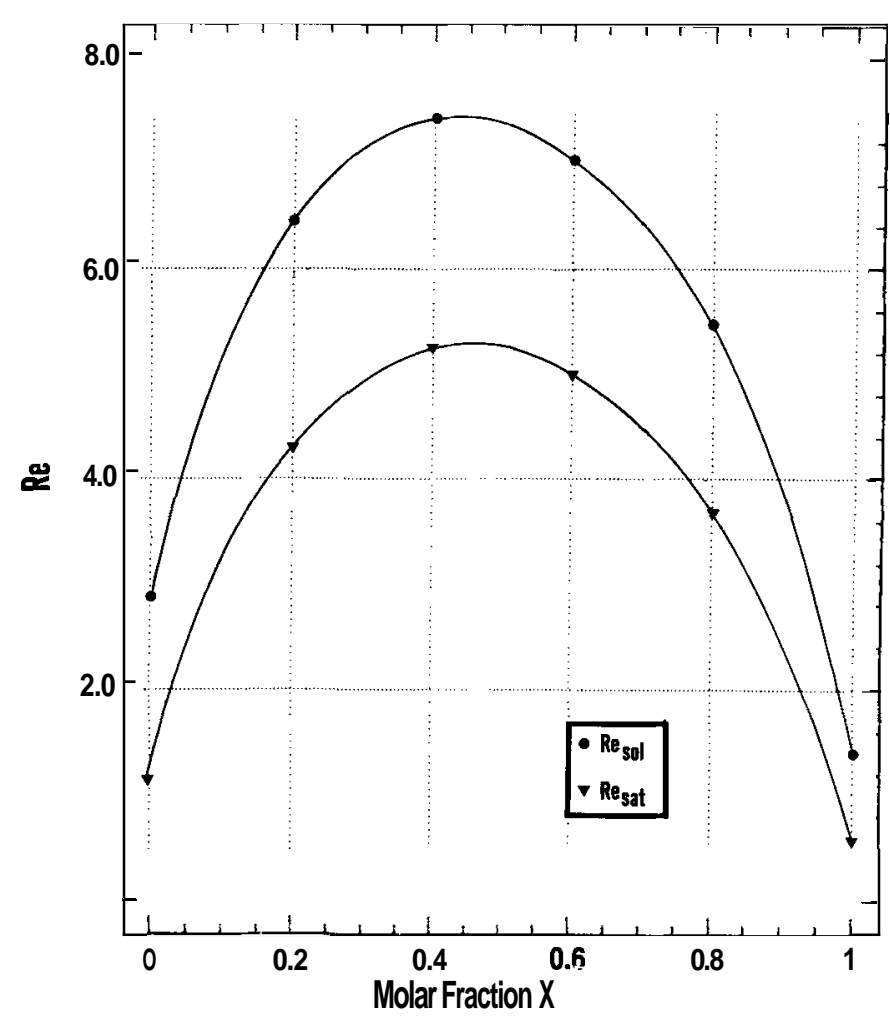

FIC. 4. Effective molar ratios $R e_{s a t}$ and $R e_{s o l}$ corresponding to the interaction of neutral liposomes with the mixed surtactant systems $\mathrm{N}$-dodecyl-N,N dimethylbetaine/sodium dodecyl sulfate vs. the molar fraction ot the zwitterionic surfactant. equilibrium involves two essential transitional steps of this interaction: the saturation of bilayers by the surfactant systems and the complete solubilization of liposomes.

Comparing the $\mathrm{Re}$ and $\mathrm{K}$ parameters with the $\mathrm{CMC}$ values (Table 1). it is interesting to note that the values display contrary tendencies, showing minimum $\mathrm{CMC}$ and maximum $\mathrm{Re}$ and $\mathrm{K}$ values for the same molar fraction $\left(X_{z \text { witter }}\right)=0.40$. From these results, it appears that the ability of each surfactant mixed system to saturate or solubilize liposomes is directly related to its CMC. These findings support the hypothesis that the solubilizing capacity of each single surfactant or surfactant mixed system depends on the concentration of active surfactant molecules in the aqueous medium capable of interacting with the PL structures; (i.e., surfactant monomers or "ion pairs"). The synergism, which decreases the CMC of the surfactant mixed system, also decreases the concentration of single surfactant monomers or ion pairs in the aqueous medium. As a consequence, this correlation results in a decrease in the capacity of these systems to solubilize liposomes. These results may explain the decrease in the irritation potential of these systems with respect to the irritation potential for the single anionic surfactant.

In the light of our results, $X_{z w i t t e r}$ appears to be a mandatory parameter that regulates the physicochemical properties of these binary systems. The specific properties of these mixtures have also been reported in different studies on viscosity, NMR line widths and solubilization of water-insoluble dyes, in which the mixed systems exhibit maxima for $X_{z \text { witter }}=0.4$ (12). 


\section{ACKNOWLEDCMENTS}

This work was supported by funds from DGICYT (Dirección General de Investigación Cientifica y Técnica) (Prog. no. PB91-0065), Spain. The authors acknowledge the expert technical assistance of G. von Knorring.

\section{REFERENCES}

1. Helenius, A., and K. Simons, Biochim. Biophys. Acta 415:29 (1975).

2. Lichtenberg. D.. R.J. Robson and E.A. Dennis. Ibid. 737:285 (1983).

3. Keren-Zur, M., M. Beigel and A. Loyter, Ibid. 983:253 (1989).

4. Miquel, M.G., O. Eidelman, M. Ollivon and A. Walter, Biochem. 28:8921 (1989).

5. Levy, D., A. Gulik. M. Seigneuret and J.L. Rigaud. Ihid. 29:9480 (1990).

6. Almog, S., B.J. Litman, W. Wimley, J. Cohen. E.J. Wachtel, Y. Barenholz. A. Ben-Shaul and D. Lichtenberg, Ibid. 29:4582 (1990).

7. Edwards, K., and M. Almgren, J. Colloid Interface Sci. 147:1 (1991).

8. Inoue, T.. T. Yamahata and R. Shimozawa, Ihid. 149:345 (1992).

9. Kragh Hansen, U., M. le Marie, J.P. Nöel, T. Gulik-Krzywicki and J.V. Møller, Biochem. 32:1648 (1993).

10. Lichtenberg, D.. Biochim. Biophvs. Acta 82 I:470 (1985).

11. Urbaneja, M.A., A. Alonso, J.M. González-Mañas, F.M. Gofii, M.A. Partearroyo, M. Tribout and S. Paredes, Biochem. J. 270:305 (1990).

12. Iwasaki, T., M. Ogawa, K. Esumi and K. Meguro, Langmuir 7:30 (1991).

13. Rosen, M.L., Ibid. 7:885 (1991).

14. Abe. M., K. Kato and K. Ogino, J. Colloid Interface Sci. 127:328 (1989).

15. Jansson, M., P. Linse and R. Rymden. J. Phys. Chem. $92: 6689$ (1988).

16. Cooper. E.R., and B. Berner, in Surfactant in Cosmetics, Surfactant Science Series, Vol. 16, edited by M.M. Rieger. Marcel Dekker Inc., New York, 1985, pp. 195-211.
17. Domínguez, G.. J.F. Balaguer, J.L. Parra and C.M. Pelejero, Int. J. Cosm. Sci. 3:57 (1981).

18. Faucher, J.A., and E.D. Goddard, J. Soc. Cosm. Chem. 29:323 (1978).

19. de la Maza, A., and J.L. Parra, J. Am. Oil Chem. Soc. 70:685 (1993).

20. de la Maza, A., and J.L. Parra, Ibid. 70:699 (1993).

21. Singleton, W.S.. M.S. Gray, M.L. Brown and J.L. White, Ibid. 42:53 (1965).

22. Rosen, M.J., J. Colloid Interface Sci. 79:587 (1981).

23. Paternostre, M.T., M. Roux and J.L. Rigaud. Biochem. 27:2668 (1988).

24. Rigaud, J.L., M.T. Paternostre and A. Bluzat, Ibid. 27:2677 (1988).

25. Szoka, F., and D. Papahadjopoulos, Liposomes: Preparation and Characterization, edited by C.G. Knight. Elsevier, Amsterdam, 1981, Chapter 3.

26. Allen, T.M., Liposome Technology, Vol. I, edited by G. Gregoriadis. CRC Press, Boca Raton, 1986, Chapter 8.

27. Mayer, L.D., M.J. Hope and P.R. Cullis, Biochim. Biophys. Acta 858: 161 (1986).

28. Ackman, R.G., C.A. Mc Leod and A.K. Banerjee, J. of Planar Chrom. 3:450 (1990).

29. Goñi, F.M., M.A. Urbaneja, J.L.R. Arrondo, A. Alonso, A.A. Durrani and D. Chapman, Eur. J. Biochem. 160:659 (1986).

30. Tanford, C., in The Hydrophobic Effect: Formation of Micelles and Biological Membranes. Wiley and Sons, New York, 1980.

31. Hall, D.G., in Nonionic Surfactants. Physical Chemistry, Surfactant Science Series, Vol. 23. edited by M.J. Schick, Marcel Dekker, New York, 1987, Chapter 5.

32. Schurtenberger, P., N. Mazer and W. Känzig, J. Phys. Chem. 89: 1042 (1985).

33. Ogino, K., M. Abe, K. Kato and R. Sakama, J. Jpn. Oil Chem. Soc. 36: 129 (1987).

[Received June 16, 1994; accepted September 15, 1994] 